\title{
Precise Point Positioning with Multipath Estimation
}

\author{
Patrick Henkel \\ Technische Universität München \\ Electrical and Computer Engineering \\ 80333 Munich, Germany \\ Email: patrick.henkel@tum.de
}

\author{
Michele Iafrancesco and Andreas Sperl \\ Advanced Navigation Solutions - ANavS GmbH \\ Precise Position and Attitude Determination Systems \\ 80798 Munich, Germany \\ Email: \{michele.iafrancesco, andreas.sperl\}@anavs.de
}

\begin{abstract}
Precise Point Positioning with low-cost GNSS receivers is attractive for numerous applications, as it can provide centimeter-level positioning without the exchange of raw measurements from a reference station. However, pseudorange multipath has to be accurately considered for a reliable ambiguity fixing. In this paper, we derive a precise model for the pseudorange multipath from the received signal, the correlator and discriminator function. We consider multiple reflections with individual amplitudes, phasings, Doppler shifts and code delays.

We estimate a pseudorange multipath parameter for each satellite in our precise position solution to exploit the temporal correlation of multipath and to prevent a mapping of multipath to other state parameters. The method was tested with real measurements from a low-cost receiver and SSR corrections and showed that a millimeter-level positioning accuracy is achievable.
\end{abstract}

\section{INTRODUCTION}

Precise Point Positioning (PPP) is very attractive for autonomous driving of vehicles as it does not require raw measurements from a reference station. However, precise corrections for atmospheric delays, satellite position and clock errors, and satellite biases are needed for a fast convergence of the Kalman filter. Code multipath and a reliable ambiguity resolution with low-cost GNSS receivers and antennas are still challenging.

The ambiguity fixing is traditionally performed in two steps: The first one includes the calculation of a float solution and the second one includes the mapping of the float solution to an integer one. In the integer least-squares estimation (LAMBDA method) of Teunissen [1], the fixing depends only on the float ambiguities and the float ambiguity covariance matrix. There are two inherent problems: First, an integer error of the float ambiguities can not be recognized from the ambiguity residuals and will result in the same integer error of the fixed solution. Secondly, the float ambiguity covariance matrix could be itself erroneous due to unmodeled or inaccurately modeled multipath. An erroneous covariance matrix results in a distorted search space, which might miss the correct integers.

Henkel and Iafrancesco [2] estimate a code multipath parameter for each satellite-satellite single difference measurement to fully exploit the temporal correlation of multipath and to prevent a mapping of the code multipath into other state parameters. The fixing method of Henkel and Sperl [3] includes two phases: the first phase is an integer collection phase and the second phase is an integer tracking phase. In the collection phase, sets of integer candidate vectors are determined based on the float solution at multiple epochs. Thereby, different float solutions are considered to increase the likelihood of including the correct candidate vector in the set of candidate vectors.

In the integer tracking phase, a single-epoch phase-only least-squares position estimate is determined for each candidate vector. The sum of squared fixed phase measurement residuals are accumulated over time. For all erroneous candidate vectors, the sum of squared residuals is drifting over time as the residuals are affected by the projection of the integer error on the orthogonal space of the geometry matrix, which depends on the time-dependent line of sight vector between the satellite and receiver. For the correct candidate vector, the residuals only include projected phase noise and multipath and, thus, are not drifting over time. An integer candidate is selected once there is a sufficient discrimination, i.e. once the position drift of one candidate is at least two times lower than the drift of any other candidate.

In this paper, we derive a precise model for the code multipath from the received signal using correlator and discriminator functions. The obtained model is integrated into a precise point positioning solution. We applied the method to real measurements from low-cost u-blox LEA 6T GPS receivers and show that a precise point positioning with precise multipath estimation is feasible.

\section{Modeling OF PSEUdorAnge ERROR DUE TO MULTIPATH}

In this section, the pseudorange error due to multipath is derived for an Early-minus-Late discriminator. We model the received signal at time $t$ as the superposition of the direct signal with amplitude $A_{0}$, code delay $\tau$, phase $\varphi$ and Doppler frequency $\omega$ and $L$ reflected signals with amplitude $A_{l}$, extra path delay $\Delta t_{l}$, phase shift $\Delta \varphi_{l}$ and Doppler offset $\Delta \omega_{l}$, i.e.

$$
\begin{aligned}
r(t)= & A_{0} \cdot c(t-\tau) \cdot e^{j(\varphi+\omega(t-\tau))} \\
& +\sum_{l=1}^{L} A_{l} \cdot c\left(t-\left(\tau+\Delta t_{l}\right)\right) \\
& \cdot e^{j\left(\varphi+\Delta \varphi_{l}+\left(\omega+\Delta \omega_{l}\right)\left(t-\left(\tau+\Delta t_{l}\right)\right)\right)}+\eta(t),
\end{aligned}
$$


with $c(t)$ being the chip pulse and $\eta$ being the noise of the received signal. We assume that all tracking loops (DLL, FLL and PLL) are in lock. In this case, we can remove the carrier from the received signal, i.e.

$$
\begin{aligned}
r^{*}(t)= & r(t) \cdot e^{-j(\varphi+\omega(t-\tau))} \\
= & A_{0} \cdot c(t-\tau)+\sum_{l=1}^{L} A_{l} \cdot c\left(t-\left(\tau+\Delta t_{l}\right)\right) \\
& \cdot e^{j\left(\Delta \varphi_{l}-\omega \Delta t_{l}-\Delta \omega_{l} \cdot\left(t-\left(\tau+\Delta t_{l}\right)\right)\right)}+\eta(t) .
\end{aligned}
$$

As the signal of interest is only in the real part of $r^{*}(t)$, we can eliminate the imaginary part of the reflected signals by a projection onto the space of real-valued numbers, i.e.

$$
\begin{aligned}
\operatorname{Re}\left\{r^{*}(t)\right\}= & A_{0} \cdot c(t-\tau) \\
& +\sum_{l=1}^{L} A_{l} \cdot c\left(t-\left(\tau+\Delta t_{l}\right)\right) \\
& \cdot \cos \left(\Delta \varphi_{l}-\omega \Delta t_{l}-\Delta \omega_{l} \cdot\left(t-\left(\tau+\Delta t_{l}\right)\right)\right) \\
& +\eta(t) .
\end{aligned}
$$

The GNSS receiver correlates $\operatorname{Re}\left\{r^{*}(t)\right\}$ with the receivergenerated signal $A_{0} \cdot c(t-\tau-\Delta)$ and obtains as correlation result:

$$
C(\Delta \tau)=\int_{0}^{T_{\mathrm{c}}} \operatorname{Re}\left\{r^{*}(t)\right\} \cdot A_{0} \cdot c(t-\tau-\Delta \tau) d(t-\tau)
$$

Let us assume that $c(t-\tau)$ is defined as a rectangular pulse, i.e.

$$
c(t-\tau)=\left\{\begin{array}{ll}
1 & \text { if } 0<t-\tau<T_{\mathrm{c}} \\
0 & \text { else. }
\end{array},\right.
$$

and that the extra-path delays $\Delta t_{l}$ are in the interval $\left[0, T_{\mathrm{C}}\right]$ for all $l$. The correlation result of Eq. (4) can then be further developed as

$$
\begin{gathered}
C(\Delta \tau)=A_{0}^{2} \cdot \int_{\max (0, \Delta \tau)}^{\min \left(\Delta \tau+T_{\mathrm{c}}, T_{\mathrm{c}}\right)} d(t-\tau) \cdot \delta_{\left(\delta \tau<T_{\mathrm{c}}\right)} \\
+\sum_{l=1}^{L} A_{0} A_{l} \cdot I_{l}\left(\min \left(\max \left(\Delta \tau, \Delta t_{l}\right), \Delta t_{l}+T_{\mathrm{c}}\right),\right. \\
\min \left(T_{\mathrm{c}}+\Delta \tau, T_{\mathrm{c}}+\Delta t_{l}\right), \\
\left.\Delta \varphi_{l}, \Delta \omega_{l}, \Delta t_{l}\right)+\tilde{\eta}(t),
\end{gathered}
$$

with $I_{l}$ being defined as

$$
\begin{aligned}
& I_{l}\left(\alpha_{l}, \beta_{l}, \Delta \varphi_{l}, \Delta \omega_{l}, \Delta t_{l}\right) \\
& =\int_{\alpha_{l}}^{\beta_{l}} \cos \left(\Delta \varphi_{l}-\omega \Delta t_{l}-\Delta \omega_{l} \cdot\left(t-\tau-\Delta t_{l}\right)\right) d(t-\tau) \\
& \quad \cdot \delta_{\left(\beta_{l}>\alpha_{l}\right)}+\tilde{\eta}(t),
\end{aligned}
$$

and

$$
\delta_{\left(\beta_{l}>\alpha_{l}\right)}= \begin{cases}1 & \text { if } \beta_{l}>\alpha_{l} \\ 0 & \text { else. }\end{cases}
$$

The slope of the correlation result is changing abruptly at some code offsets $\Delta \tau$. The set of characteristic points is given by

$$
\left\{-T_{\mathrm{c}},-T_{\mathrm{c}}+\Delta t_{l}, 0, \Delta t_{l}, T_{\mathrm{c}}, T_{\mathrm{c}}+\Delta t_{l}\right\}, \quad l \in\{1, \ldots, L\} .
$$

The correlation result at these characteristic points can be derived as

$$
\begin{aligned}
& C\left(-T_{\mathrm{c}}\right)=0 \\
& C\left(-T_{\mathrm{c}}+\Delta t_{l}\right) \\
& =A_{0}^{2} \Delta t_{l}+\sum_{l^{\prime}=1}^{L} A_{0} A_{l^{\prime}} \cdot I_{l^{\prime}}\left(\Delta t_{l^{\prime}}, \Delta t_{l}, \Delta \varphi_{l^{\prime}}, \Delta \omega_{l^{\prime}}, \Delta t_{l^{\prime}}\right) \\
& C(0) \\
& =A_{0}^{2} T_{\mathrm{c}}+\sum_{l^{\prime}=1}^{L} A_{0} A_{l^{\prime}} \cdot I_{l^{\prime}}\left(\Delta t_{l^{\prime}}, T_{\mathrm{c}}, \Delta \varphi_{l^{\prime}}, \Delta \omega_{l^{\prime}}, \Delta t_{l^{\prime}}\right) \\
& \left.C\left(\Delta t_{l}\right) \quad \min \left(T_{\mathrm{c}}+\Delta t_{l}, T_{\mathrm{c}}+\Delta t_{l^{\prime}}\right), \Delta \varphi_{l^{\prime}}, \Delta \omega_{l^{\prime}}, \Delta t_{l^{\prime}}\right) \\
& =A_{0}^{2}\left(T_{\mathrm{c}}-\Delta t_{l}\right)+\sum_{l^{\prime}=1}^{L} A_{0} A_{l^{\prime}} \cdot I_{l^{\prime}}\left(\max \left(\Delta t_{l}, \Delta t_{l^{\prime}}\right)\right. \\
& C\left(T_{\mathrm{c}}\right) \\
& =\sum_{l^{\prime}=1}^{L} A_{0} A_{l^{\prime}} \cdot I_{l^{\prime}}\left(T_{\mathrm{c}}, T_{\mathrm{c}}+\Delta t_{l^{\prime}}, \Delta \varphi_{l^{\prime}}, \Delta \omega_{l^{\prime}}, \Delta t_{l^{\prime}}\right) \\
& C\left(T_{\mathrm{c}}+\Delta t_{l}\right) \\
& =\sum_{l^{\prime}=1}^{L} A_{0} A_{l^{\prime}} \cdot I_{l^{\prime}}\left(\min _{\mathrm{c}}+\Delta t_{l^{\prime}}, \Delta \varphi_{\mathrm{c}}+\Delta t_{l}, T_{\mathrm{c}}+\Delta \omega_{l^{\prime}}, \Delta t_{l^{\prime}}\right) \\
& \left.\quad t_{l^{\prime}}\right)
\end{aligned}
$$

GNSS receivers use a discriminator for tracking the code delay $\Delta \tau$. The Early-minus-Late discriminator [4] is widely used and defined as

$$
\begin{aligned}
D(\Delta \tau) & =C_{\mathrm{E}}(\Delta \tau)-C_{\mathrm{L}}(\Delta \tau) \\
& =C\left(\Delta \tau-d T_{\mathrm{c}} / 2\right)-C\left(\Delta \tau+d T_{\mathrm{c}} / 2\right)
\end{aligned}
$$

with $d T_{\mathrm{c}} / 2, d \leq 1$, being the correlator spacing. We assume without loss of generality that the extra-path delay $\Delta t_{l}$ is smaller than $d T_{\mathrm{c}} / 2$. The discriminator function is continuous in the interval

$$
\Delta \tau \in\left[-\frac{d T_{\mathrm{c}}}{2}+\max _{l}\left(\Delta t_{l}\right), \frac{d T_{\mathrm{c}}}{2}\right] .
$$

We denote the discriminator function at the lower and upper end of this interval by $D_{\mathrm{l}}$ and $D_{\mathrm{u}}$, i.e.

$$
\begin{aligned}
D_{\mathrm{l}} & =C\left(-d T_{\mathrm{c}}+\max _{l}\left(\Delta t_{l}\right)\right)-C\left(\max _{l}\left(\Delta t_{l}\right)\right) \\
D_{\mathrm{u}} & =C(0)-C\left(d T_{\mathrm{c}}\right),
\end{aligned}
$$

where the correlator results can be obtained by linear interpolation using the correlation results at the characteristic points of Eq. (10)-(14). The discriminator function has a zero crossing in this interval. The non-zero code delay at the zerocrossing represents the pseudorange error due to multipath. We approximate the discriminator function by a linear function, i.e.

$$
D(\Delta \tau)=D_{\mathrm{l}}+\frac{D_{\mathrm{u}}-D_{\mathrm{l}}}{\Delta \tau_{\mathrm{u}}-\Delta \tau_{\mathrm{l}}} \cdot\left(\Delta \tau-\Delta \tau_{\mathrm{l}}\right)
$$


The pseudorange error due to multipath is obtained by setting $D(\Delta \tau)=0$ and by solving it for $\Delta \tau$, i.e.

$$
\Delta \tau_{\mathrm{MP}}=\Delta \tau_{\mathrm{l}}-\frac{D_{\mathrm{l}}}{D_{\mathrm{u}}-D_{\mathrm{l}}} \cdot\left(\Delta \tau_{\mathrm{u}}-\Delta \tau_{\mathrm{l}}\right) .
$$

Fig. 1 includes a graphical representation of the multipathinduced pseudorange error for a simplified case with $L=1$, $\Delta \varphi_{1}=0$ and $\Delta \omega_{1}=0$. In this special case, the pseudorange error due to multipath simplifies to

$$
\begin{aligned}
\Delta \rho_{\mathrm{MP}} & =c \Delta \tau_{\mathrm{MP}} \\
& =\frac{A_{1}}{A_{0}+A_{1}} \cdot \Delta t_{1} \quad \forall \quad \Delta t_{1} \leq \frac{d T_{\mathrm{c}}}{2}, d \leq 1,
\end{aligned}
$$

which is directly proportional to both the amplitude $A_{1}$ and extra path delay $\Delta t_{1}$ of the reflected signal but is independent from the correlator spacing $d$.
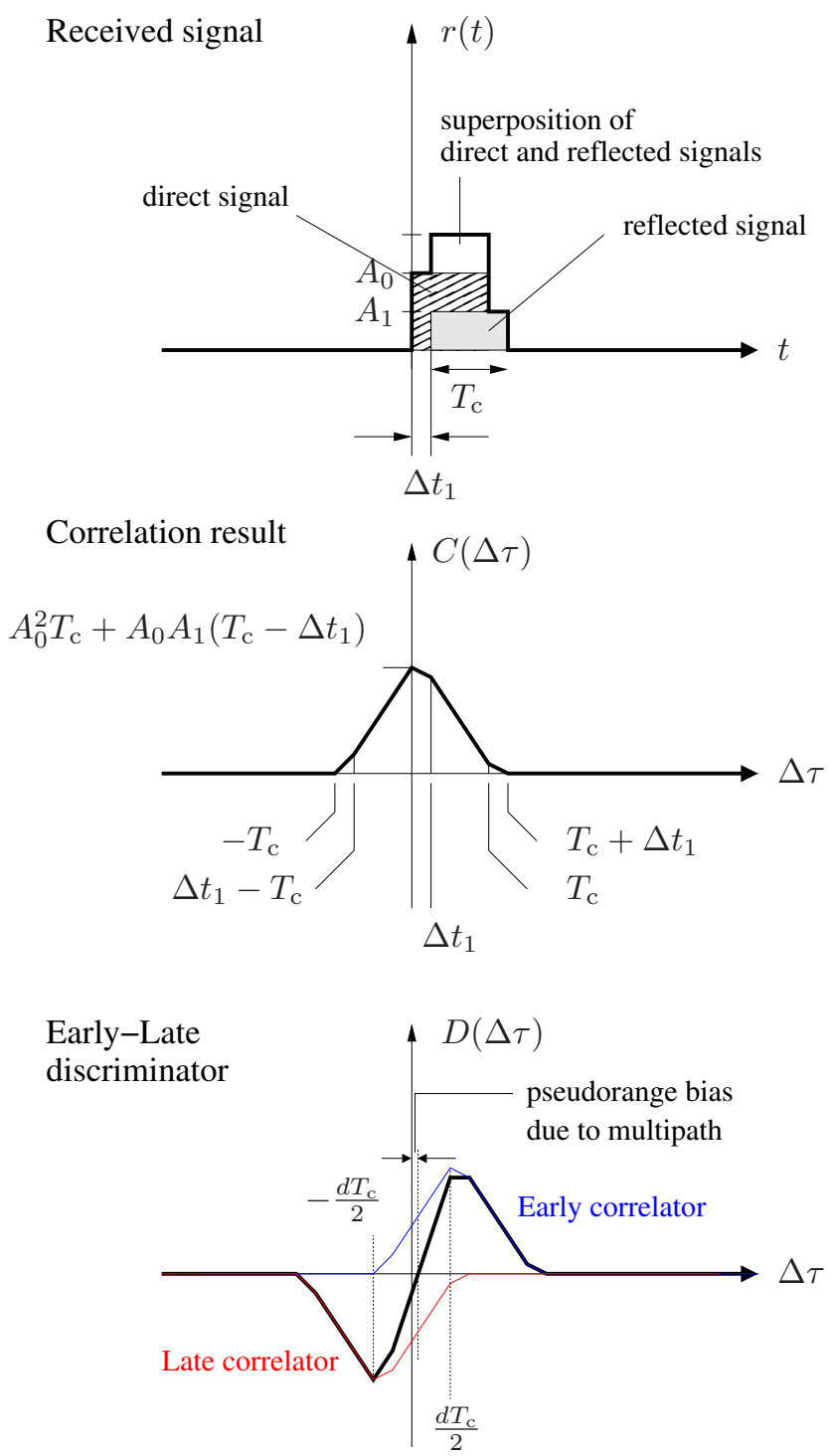

Fig. 1: Early-Late discriminator result with multipath

For a more realistic multipath analysis, one has to consider the amplitude $A_{l}$, the phase shift $\Delta \varphi_{l}$, the Doppler offset $\Delta \omega_{l}$ and the extra-path delay $\Delta t_{l}$ for $l=\{1, \ldots, L\}$ reflections. We assume a random walk process for each parameter, i.e.

$$
\left\{\begin{array}{c}
A_{l}\left(t_{n}\right) \\
\Delta \varphi_{l}\left(t_{n}\right) \\
\Delta \omega_{l}\left(t_{n}\right) \\
\Delta t_{l}\left(t_{n}\right)
\end{array}\right\}=\left\{\begin{array}{c}
\left|A_{l}\left(t_{n-1}\right)+\varepsilon_{A_{l}}\left(t_{n}\right)\right| \\
\Delta \varphi_{l}\left(t_{n-1}\right)+\varepsilon_{\Delta \varphi_{l}}\left(t_{n}\right) \\
\Delta \omega_{l}\left(t_{n-1}\right)+\varepsilon_{\Delta \omega_{l}}\left(t_{n}\right) \\
\left|\Delta t_{l}\left(t_{n-1}\right)+\varepsilon_{\Delta t_{l}}\left(t_{n}\right)\right|
\end{array}\right\},
$$

where the absolute value is considered for $A_{l}$ and $\Delta t_{l}$ as both the amplitude and extra path delay are non-negative.

\section{Precise Point Positioning}

We use satellite-satellite single difference (SD) measurements to eliminate receiver dependent biases and clock offsets. The SD carrier phases are modeled as

$$
\begin{aligned}
\lambda \varphi_{r}^{k l}= & \vec{e}_{r}^{k}\left(\vec{x}_{r}-\vec{x}^{k}\right)-\vec{e}_{r}^{l}\left(\vec{x}_{r}-\vec{x}^{l}\right) \\
& +\vec{e}_{r}^{k l} \Delta \vec{x}_{\mathrm{ET}}+\lambda \Delta \varphi_{\mathrm{PCO}}^{k l}+\lambda \Delta \varphi_{\mathrm{PW}}^{k l} \\
& -c \delta \tau^{k l}+T_{r}^{k l}-I_{r}^{k l}+\lambda N_{r}^{k l}+\lambda / \Delta N_{r}^{k l} \\
& +\beta^{k l}+\varepsilon_{\varphi_{r}^{k l}},
\end{aligned}
$$

with the satellite-receiver line of sight vector $\vec{e}_{r}^{k}$, the receiver position $\vec{x}_{r}$, the satellite position $\vec{x}^{k}$, the earth tide $\Delta \vec{x}_{\mathrm{ET}}$, the wavelength $\lambda$, the SD phase center offset $\Delta \varphi_{\mathrm{PCO}}^{k l}$, the SD phase wind-up $\Delta \varphi_{\mathrm{PW}}^{k l}$, the speed of light $c$, the satellite clock offset $\delta \tau^{k}$, the tropospheric delay $T_{r}^{k}$, the ionospheric delay $I_{r}^{k}$, the integer ambiguity $N_{r}^{k}$, the number of half cycle slips $\Delta N_{r}^{k l}$, the satellite phase bias $\beta^{k}$ and the phase noise $\varepsilon_{\varphi_{r}^{k l}}$. The SD pseudoranges are modeled as

$$
\begin{aligned}
\rho_{r}^{k l}= & \vec{e}_{r}^{k}\left(\vec{x}_{r}-\vec{x}^{k}\right)-\vec{e}_{r}^{l}\left(\vec{x}_{r}-\vec{x}^{l}\right)+\vec{e}_{r}^{k l} \Delta \vec{x}_{\mathrm{ET}} \\
& -c \delta \tau^{k l}+T_{r}^{k l}+I_{r}^{k l}+\Delta \rho_{\mathrm{MP}_{r}}^{k l}+b^{k l}+\varepsilon_{\eta_{r}^{k l}},
\end{aligned}
$$

with the code multipath pseudorange error $\Delta \rho_{\mathrm{MP}_{r}}^{k}$ based on Eq. (19), the satellite code bias $b^{k}$ and the code noise $\varepsilon_{\eta_{r}^{k}}$. The third GNSS observable is the Doppler measurement being modeled as

$$
\begin{aligned}
\tilde{f}_{\mathrm{D}, r}^{k l}= & -f_{\mathrm{c}}\left(\vec{e}_{r}^{k}\left(\dot{\vec{x}}_{r}-\dot{\vec{x}}^{k}\right) / c-\vec{e}_{r}^{l}\left(\dot{\vec{x}}_{r}-\dot{\vec{x}}^{l}\right) / c\right) \\
& -f_{\mathrm{c}} \delta \dot{\tau}^{k l}+\varepsilon_{f_{\mathrm{D}_{r}}^{k l}},
\end{aligned}
$$

with the carrier frequency $f_{\mathrm{c}}$, the receiver velocity $\dot{\vec{x}}_{r}$, the satellite velocity $\dot{\vec{x}}^{k}$, the satellite clock drift $\delta \dot{\tau}^{k}$ and the Doppler measurement noise $\varepsilon_{f_{\mathrm{D}_{r}}^{k}}$.

We subtract the a priori known satellite positions, satellite clock offsets, tropospheric/ ionospheric delays, satellite phase/ code bias estimates, Earth tides, phase center offsets and phase wind up using precise models from Eq. (22) and (23) to obtain

$$
\begin{aligned}
\lambda \tilde{\varphi}_{r}^{k l}= & \lambda \varphi_{r}^{k l}+\vec{e}_{r}^{k} \vec{x}^{k}-\vec{e}_{r}^{l} \vec{x}^{l}+c \delta \tau^{k l} \\
& -\hat{T}_{r}^{k l}+\hat{I}_{r}^{k l}-\hat{\beta}^{k l} \\
& -\vec{e}_{r}^{k l} \Delta \vec{x}_{\mathrm{ET}}-\lambda \Delta \varphi_{\mathrm{PCO}}^{k l}-\lambda \Delta \varphi_{\mathrm{PW}}^{k l} \\
= & \left(\vec{e}_{r}^{k}-\vec{e}_{r}^{l}\right) \vec{x}_{r}+\lambda N_{r}^{k l}+\lambda / 2 \Delta N_{r}^{k l} \\
& +\Delta T_{r}^{k l}-\Delta I_{r}^{k l}+\varepsilon_{\varphi_{r}^{k l}},
\end{aligned}
$$


and

$$
\begin{aligned}
\tilde{\rho}_{r}^{k l}= & \rho_{r}^{k l}+\vec{e}_{r}^{k} \vec{x}^{k}-\vec{e}_{r}^{l} \vec{x}^{l}+c \delta \tau^{k l} \\
& -\hat{T}_{r}^{k l}-\hat{I}_{r}^{k l}-\hat{b}^{k l}-\vec{e}_{r}^{k l} \Delta \vec{x}_{\mathrm{ET}} \\
= & \left(\vec{e}_{r}^{k}-\vec{e}_{r}^{l}\right) \vec{x}_{r}+\Delta T_{r}^{k l}+\Delta I_{r}^{k l}+\Delta \rho_{\mathrm{MP}_{r}}^{k l}+\varepsilon_{\eta_{r}^{k l}} .
\end{aligned}
$$

For the Doppler measurement, we obtain similarly

$$
\begin{aligned}
\tilde{f}_{\mathrm{D}, r}^{k l} & =f_{\mathrm{D}, r}^{k l}-f_{\mathrm{c}}\left(\vec{e}_{r}^{k} \dot{\vec{x}}^{k}-\vec{e}_{r}^{l} \dot{\vec{x}}^{l}\right) / c+f_{\mathrm{c}} \delta \dot{\tau}^{k l} \\
& =-f_{\mathrm{c}}\left(\vec{e}_{r}^{k}-\vec{e}_{r}^{l}\right) \dot{\vec{x}}_{r} / c
\end{aligned}
$$

If $\left\{\Delta T_{r}^{k l}, \Delta I_{r}^{k l}\right\} \ll \lambda / 2$, the residual atmospheric errors can be neglected and the SD ambiguities $N_{r}^{k l}$ can be considered as integer valued. A Kalman filter is used to estimate the absolute position $\vec{x}_{r}$, the SD ambiguities $N_{r}^{k l}$ and the code multipath pseudorange errors $\Delta \rho_{\mathrm{MP}_{r}}^{k l}$. If the residual atmospheric errors are not negligible, they are implicitly mapped to the ambiguities and code multipath pseudorange errors. In this case, the ambiguities are no longer integer valued.

\section{CYCLE SLIP CORRECTION}

The cycle slips are corrected in a pre-processing. The timedifference between the SD phase measurements of two subsequent epochs eliminates the constant integer ambiguity term but does not cancel the position term for moving receivers. Therefore, we subtract the time difference of the position term $\vec{e}_{r}^{k l} \vec{x}_{r}$ from the time-differenced SD phase measurements and obtain from Eq. (25)

$$
\begin{aligned}
& \lambda \tilde{\varphi}_{r}^{k l}\left(t_{n}\right)-\lambda \tilde{\varphi}_{r}^{k l}\left(t_{n-1}\right) \\
& -\left(\vec{e}_{r}^{k l}\left(t_{n}\right) \hat{\vec{x}}_{\text {pred }, r}\left(t_{n}\right)-\vec{e}_{r}^{k l}\left(t_{n-1}\right) \check{\vec{x}}_{r}\left(t_{n-1}\right)\right) \\
& =\lambda / 2 \Delta N_{r}^{k l}+\left(\vec{e}_{r}^{k l}\left(t_{n}\right) \varepsilon_{\hat{\vec{x}}_{\text {pred }, r}}\left(t_{n}\right)-\vec{e}_{r}^{k l}\left(t_{n-1}\right) \varepsilon_{\check{\vec{x}}_{r}}\left(t_{n-1}\right)\right) \\
& \quad+\varepsilon_{\varphi_{r}^{k l}}\left(t_{n}\right)-\varepsilon_{\varphi_{r}^{k k}}\left(t_{n-1}\right),
\end{aligned}
$$

which leaves the cycle slips, the errors of the position estimates and the phase noise as unknowns. As the cycle slip detection is typically performed in a pre-processing before positioning, the position of the current ( $n$-th) epoch must be predicted. We integrate the position by using the acceleration measurement $a^{\mathrm{b}}$, which is given in the body-fixed frame and affected by the accelerometer bias $b_{\mathrm{a}}^{\mathrm{b}}$ and the gravity vector $g^{\mathrm{b}}$. Applying the respective corrections and rotations enables us to predict the position as

$$
\begin{aligned}
& \hat{\vec{x}}_{\text {pred }, r}\left(t_{n}\right)=\hat{\vec{x}}_{r}\left(t_{n-1}\right)+\dot{\overrightarrow{\vec{x}}}_{r}\left(t_{n-1}\right) \cdot\left(t_{n}-t_{n-1}\right) \\
& +\int_{t_{n-1}}^{t_{n}} \int_{t_{n-1}}^{t_{n}}\left(R_{\mathrm{n}}^{\mathrm{e}}(t) R_{\mathrm{b}}^{\mathrm{n}}(t)\left(a^{\mathrm{b}}(t)-b_{\mathrm{a}}^{\mathrm{b}}(t)\right)-R_{\mathrm{n}}^{\mathrm{e}}(t) g^{\mathrm{n}}\right) d^{2} t .
\end{aligned}
$$

The accuracy of the predicted position and, thus, the reliability of cycle slip correction is mainly limited by an imperfect knowledge of the attitude-dependant rotation matrix $R_{\mathrm{b}}^{\mathrm{n}}$ and acceleration bias $a^{\mathrm{b}}$. The cycle slip correction can be further enhanced by using a tree search including all satellites and by using baseline length a priori information as described by Henkel and Oku in [5].

\section{Joint Precise Point Positioning AND ATTITUDE DETERMINATION}

We consider $r$ GNSS receivers/ antennas for determining both the absolute position and the three-dimensional attitude as introduced by Teunissen in [6] as Array-aided Precise Point Positioning. The absolute position of the $r$-th receiver is expressed in terms of the absolute position $\vec{x}_{1}$ of the first receiver and the baseline vector $\vec{b}_{1 r}$, i.e.

$$
\vec{x}_{r}=\vec{x}_{1}-\vec{b}_{1 r} \quad \forall \quad r \geq 2 .
$$

Similarly, the SD ambiguities of the $r$-th receiver can be related to the SD ambiguities of the first receiver using the double difference ambiguities $N_{1 r}^{k l}$, i.e.

$$
N_{r}^{k l}=N_{1}^{k l}-N_{1 r}^{k l} \quad \forall \quad r \geq 2 .
$$

The estimation of $N_{1}^{k l}$ and $N_{1 r}^{k l}$ instead of $N_{r}^{k l}$ is advantageous as the double difference ambiguities of the attitude baseline are always integer valued while $N_{r}^{k l}$ might not necessarily be integer valued. The attitude baseline vectors can be described both in a local body-fixed frame (being notated as b-frame) and in the North-East-Down (NED) navigation frame (being notated as n-frame). The transformation $R_{\mathrm{b}}^{\mathrm{n}}$ from the b-frame into the n-frame depends on the attitude, i.e. the roll angle $\varphi$, the pitch angle $\theta$ and the yaw angle $\psi$ and is given by Jekeli [7] as

$$
R_{\mathrm{b}}^{\mathrm{n}}=R_{3}(-\psi) R_{2}(-\theta) R_{1}(-\varphi),
$$

where $R_{j}$ is a rotation around the $j$-th axis. The baseline vectors of the attitude and their derivatives are given in the n-frame by

$$
\begin{aligned}
& \vec{b}_{1 r}^{\mathrm{n}}=R_{\mathrm{b}}^{\mathrm{n}} \vec{b}_{1 r}^{\mathrm{b}} \\
& \dot{\vec{b}}_{1 r}^{\mathrm{n}}=\dot{R}_{\mathrm{b}}^{\mathrm{n}} \vec{b}_{1 r}^{\mathrm{b}}+R_{\mathrm{b}}^{\mathrm{n}} \dot{\vec{b}}_{1 r}^{\mathrm{b}},
\end{aligned}
$$

with $\dot{R}_{\mathrm{b}}^{\mathrm{n}}$ being a function of the attitude Euler angles and their derivatives $\dot{\varphi}, \dot{\theta}$ and $\dot{\psi}$.

The precise estimation of the attitude with GNSS has several advantages that go clearly beyond the attitude information itself. First, the reliability of the RTK cycle slip correction is significantly improved, as the orientation of the gravity vector and, thus, the acceleration can be described more accurately. Second, the knowledge of the attitude baseline $\vec{b}_{1 r}$ and its double difference ambiguities ambiguities $N_{1 r}^{k l}$ enables us to express the SD measurements of the $r$-th receiver in terms of the SD measurements of the 1-st receiver:

$$
\begin{aligned}
\lambda\left(\tilde{\varphi}_{r}^{k l}+N_{1 r}^{k l}\right)= & \lambda \tilde{\varphi}_{1}^{k l}-\left(\vec{e}_{1}^{k}-\vec{e}_{1}^{l}\right) \vec{x}_{1} \\
& +\left(\vec{e}_{r}^{k}-\vec{e}_{r}^{l}\right)\left(\vec{x}_{1}-\vec{b}_{1 r}\right)-\varepsilon_{\varphi_{1 r}^{k l}}
\end{aligned}
$$

which increases the number of carrier phase measurements from $K$ to $R K$ without any further unknowns. Thus, the number of available carrier phase measurements from $R$ single frequency receivers corresponds to the number of carrier phase measurements of one single multi-frequency receiver with $R$ frequencies. 
The attitude ambiguity fixing is performed by an integer least-squares estimation using soft baseline length a priori information, i.e.

$$
\min _{N_{1 r}, \vec{b}_{1 r}}\left(\left\|z_{1 r}-H \vec{b}_{1 r}-A N_{1 r}\right\|_{\Sigma_{z}^{-1}}^{2}+\frac{\left(\left\|\vec{b}_{1 r}\right\|-\bar{l}_{1 r}\right)^{2}}{\sigma_{\bar{l}_{1 r}}^{2}}\right)
$$

with the baseline length a priori information $\bar{l}_{1 r}$ with standard deviation $\sigma_{\bar{l}_{1 r}}$ and the vector of double difference phase and code measurements

$$
z_{1 r}=\left(\lambda \tilde{\varphi}_{1 r}^{1 l}, \ldots, \lambda \tilde{\varphi}_{1 r}^{K l}, \tilde{\rho}_{1 r}^{1 l}, \ldots, \tilde{\rho}_{1 r}^{K l}\right)^{\mathrm{T}}
$$

and the geometry and design matrices

$$
H=1^{2 \times 1} \otimes\left(\begin{array}{c}
\vec{e}_{1}^{1 l} \\
\vdots \\
\vec{e}_{1}^{K l}
\end{array}\right) \quad A=\left(\begin{array}{c}
\lambda \cdot I^{K \times K} \\
0
\end{array}\right) .
$$

The solution to the problem of Eq. (35) is described in details by Henkel and Günther in [8] and by Teunissen in [9]. The magnitudes of the search intervals in the tree search were reduced in [10] by including soft baseline length a priori information in the bound calculation. Henkel and Kiam reduced the search space by using an a priori information on the 3D baseline vector instead of the baseline length [11].

Once the double difference ambiguities of the attitude baselines are fixed, the SD carrier phase measurements are re-adjusted and stacked with the pseudorange and Doppler measurements, i.e.

$$
\begin{aligned}
z^{\mathrm{GPS}}=( & \lambda \tilde{\varphi}_{1}^{\mathrm{T}}, \lambda\left(\tilde{\varphi}_{2}+\check{N}_{12}\right)^{\mathrm{T}} \ldots, \lambda\left(\tilde{\varphi}_{R}+\check{N}_{1 R}\right)^{\mathrm{T}}, \\
& \left.\tilde{\rho}_{1}^{\mathrm{T}}, \ldots, \tilde{\rho}_{R}^{\mathrm{T}}, \tilde{f}_{\mathrm{D}_{1}}^{\mathrm{T}}, \ldots, \tilde{f}_{\mathrm{D}_{R}}^{\mathrm{T}}\right)^{\mathrm{T}} .
\end{aligned}
$$

We also consider the inertial measurements for joint precise point positioning and attitude determination. The accelerometer provides its measurements in the body-fixed b-frame, which are modeled according to Jekeli [7] and our earlier publication, see e.g. [12], as

$$
\begin{aligned}
a^{\mathrm{b}}\left(t_{n}\right)= & R_{\mathrm{e}}^{\mathrm{b}}\left(t_{n}\right) a^{\mathrm{e}}\left(t_{n}\right)+b_{a}^{\mathrm{b}}\left(t_{n}\right)+R_{\mathrm{n}}^{\mathrm{b}}\left(\begin{array}{l}
0 \\
0 \\
g
\end{array}\right)+\varepsilon_{a}^{\mathrm{b}}\left(t_{n}\right) \\
= & R_{\mathrm{n}}^{\mathrm{b}}\left(t_{n}\right) R_{\mathrm{e}}^{\mathrm{n}}\left(t_{n}\right) a^{\mathrm{e}}\left(t_{n}\right)+b_{a}^{\mathrm{b}}\left(t_{n}\right) \\
& +g\left(\begin{array}{c}
-\sin \left(\theta\left(t_{n}\right)\right) \\
\cos \left(\theta\left(t_{n}\right)\right) \sin \left(\varphi\left(t_{n}\right)\right) \\
\cos \left(\theta\left(t_{n}\right)\right) \cos \left(\varphi\left(t_{n}\right)\right)
\end{array}\right)+\varepsilon_{a}^{\mathrm{b}}\left(t_{n}\right),(39)
\end{aligned}
$$

with the rotation matrices $R_{\mathrm{n}}^{\mathrm{b}}$ and $R_{\mathrm{e}}^{\mathrm{n}}$ for transforming the acceleration $a^{\mathrm{e}}$ from the ECEF (e-) frame into the body-fixed using the navigation (n-) frame (NED) as intermediate frame, the acceleration bias $b_{\mathrm{a}}^{\mathrm{b}}$ in the body-fixed frame, the magnitude $g$ of the gravity and the measurement noise $\varepsilon_{\mathrm{a}}^{\mathrm{b}}$. The rotation from the e-frame into the n-frame depends on the latitude $\varphi_{1}$ and longitude $\varphi_{1}$ of the receiver and is given by

$$
R_{\mathrm{e}}^{\mathrm{n}}\left(t_{n}\right)=R_{2}\left(-\pi / 2-\varphi_{1}\left(t_{n}\right)\right) R_{3}\left(\lambda_{1}\left(t_{n}\right)\right) .
$$

The rotation from the $n$-frame into the $b$-frame is given by the inverse of Eq. (32).
The gyroscope senses the angular rates $\omega_{\mathrm{ib}}^{\mathrm{b}}$ of the bodyfixed frame with respect to the inertial frame in the bodyfixed frame. These angular rates are the sum of $\omega_{\mathrm{in}}^{\mathrm{b}}, \omega_{\mathrm{nb}}^{\mathrm{b}}$, the gyroscope biases $b_{\omega_{\mathrm{ib}}}^{\mathrm{b}}$ and the measurement noise $\eta_{\omega_{\mathrm{ib}}}^{\mathrm{b}}$, and are modeled as

$$
\omega_{\mathrm{ib}}^{\mathrm{b}}\left(t_{n}\right)=R_{\mathrm{n}}^{\mathrm{b}}\left(t_{n}\right) \omega_{\mathrm{in}}^{\mathrm{n}}\left(t_{n}\right)+\omega_{\mathrm{nb}}^{\mathrm{b}}\left(t_{n}\right)+b_{\omega_{\mathrm{ib}}}^{\mathrm{b}}\left(t_{n}\right)+\eta_{\omega_{\mathrm{ib}}}^{\mathrm{b}}\left(t_{n}\right) .
$$

The first term of Eq. (41) depends on the latitude $\varphi_{1}$, the rates $\left\{\dot{\varphi}_{1}, \dot{\lambda}_{1}\right\}$ of latitude and longitude, and the Earth rotation rate $\omega_{\mathrm{e}}$, and is given by Jekeli [7] (p. 25, Eq. 1.89) as

$$
\omega_{\mathrm{in}}^{\mathrm{n}}=\left(\begin{array}{c}
\left(\dot{\lambda}_{1}+\omega_{\mathrm{e}}\right) \cos \left(\varphi_{1}\right) \\
-\dot{\varphi}_{1} \\
-\left(\dot{\lambda}_{1}+\omega_{\mathrm{e}}\right) \sin \left(\varphi_{1}\right)
\end{array}\right) .
$$

The second term of Eq. (41) can be related to the Euler angular rates (see Jekeli [7], p. 26, Eq. 1.94) as

$$
\begin{aligned}
\omega_{\mathrm{nb}}^{\mathrm{b}} & =R_{1}(\varphi) R_{2}(\theta)\left(\begin{array}{c}
0 \\
0 \\
\dot{\psi}
\end{array}\right)+R_{1}(\varphi)\left(\begin{array}{l}
0 \\
\dot{\theta} \\
0
\end{array}\right)+\left(\begin{array}{c}
\dot{\varphi} \\
0 \\
0
\end{array}\right) \\
& =\left(\begin{array}{rrr}
1 & 0 & -\sin (\theta) \\
0 & \cos (\varphi) & \cos (\theta) \sin (\varphi) \\
0 & -\sin (\varphi) & \cos (\theta) \cos (\varphi)
\end{array}\right)\left(\begin{array}{c}
\dot{\varphi} \\
\dot{\theta} \\
\dot{\psi}
\end{array}\right) \cdot
\end{aligned}
$$

The inertial measurements are stacked in the vector

$$
z^{\mathrm{INS}}=\left(\left(a^{\mathrm{b}}\right)^{\mathrm{T}},\left(\omega_{\mathrm{ib}}^{\mathrm{b}}\right)^{\mathrm{T}}\right)^{\mathrm{T}} .
$$

We use an extended Kalman filter for joint precise point positioning and attitude determination with GNSS/ INS sensor fusion. The sensor fusion is described by Henkel and Iafrancesco in [2][12] and includes the following state vector

$$
\begin{aligned}
x= & (\vec{x}_{1}, \dot{\vec{x}}_{1}, \ddot{\vec{x}}_{1}, \varphi, \theta, \psi, \dot{\varphi}, \dot{\theta}, \dot{\psi}, N_{1}^{k l}, \underbrace{N_{12}^{k l}, \ldots, N_{1 R}^{k l}}_{\mathbb{Z}}, \\
& \left.\Delta \rho_{\mathrm{MP}_{1}^{k l}}, \ldots, \Delta \rho_{\mathrm{MP}_{R}^{k l}},\left(b_{\mathrm{a}}^{\mathrm{b}}\right)^{\mathrm{T}},\left(b_{\omega_{\mathrm{ib}}}^{\mathrm{b}}\right)^{\mathrm{T}}\right) .
\end{aligned}
$$

The state vector is predicted with a linear movement model:

$$
\hat{x}_{n}^{-}=\Phi_{n} \hat{x}_{n-1}^{+},
$$

and updated by GPS and INS measurements in an alternating manner, i.e.

$$
\hat{x}_{n}^{+}=\hat{x}_{n}^{-}+K_{n}\left(z_{n}^{\mathrm{GPS} / \mathrm{INS}}-h_{n}\left(\hat{x}_{n}^{-}\right)\right),
$$

with $h_{n}\left(x_{n}\right)$ describing the non-linear relationship between $x_{n}$ and $z_{n}$. Once the state vector has sufficiently converged, the SD ambiguities $N_{1}^{k l}$ are fixed. We use the decorrelation and tree search of LAMBDA [1] for the determination of integer candidates. We select the candidate vector that has the minimum baseline drift among all candidates with sufficiently small sum of squared ambiguity residuals and sufficiently small sum of squared fixed phase measurement residuals, i.e.

$$
\begin{gathered}
\check{N}_{1}=\min _{N_{1}}\left\|\frac{\partial \check{b}_{1 r}\left(N_{1}\right)}{\partial t}\right\| \text { s.t. }\left\|\hat{N}_{1}-N_{1}\right\|_{\Sigma_{\hat{N}_{1}}^{-1}}^{2}<\chi_{N_{1}}^{2} \\
\wedge\left\|\lambda \varphi_{1}-\lambda \check{\varphi}_{1}\left(N_{1}\right)\right\|_{\Sigma_{\lambda \varphi_{1}}^{-1}}^{2}<\chi_{\varphi_{1}}^{2},
\end{gathered}
$$


where the baseline drift is determined by least-squares estimation using the linear model

$$
\vec{b}_{1 r}\left(t_{n}\right)=\vec{b}_{1 r}\left(t_{n-m}\right)+\frac{\partial \check{b}_{1 r}\left(N_{1}\right)}{\partial t} \cdot\left(t_{n}-t_{n-m}\right) .
$$

A baseline drift occurs for wrong ambiguity candidates due to the changing satellite geometry, and can be separated from the noise and phase multipath after 2 to 5 minutes. We consider an integer candidate as reliable once the baseline drift of the best candidate is at least two times lower than the baseline drift of any other candidate.

\section{Vi. Measurement Results}

This section shows the performance of multipath estimation and the achievable PPP accuracy with low-cost GPS receivers.

We used the following measurement set-up: Two patch antennas were mounted on the roof of a static car and connected to u-blox LEA 6T single frequency GPS receivers. The measurements were taken on an open-field with negligible multipath. The ionospheric and tropospheric delays as well as satellite phase and code biases were obtained from SSR corrections. Artificial multipath was generated using Eq. (19) and added to the real measurements.

Fig. 2 includes a comparison of the generated and estimated pseudorange multipaths. The enlarged sections show that the estimated multipath closely follows the generated one and that the difference is in the order of the code noise.

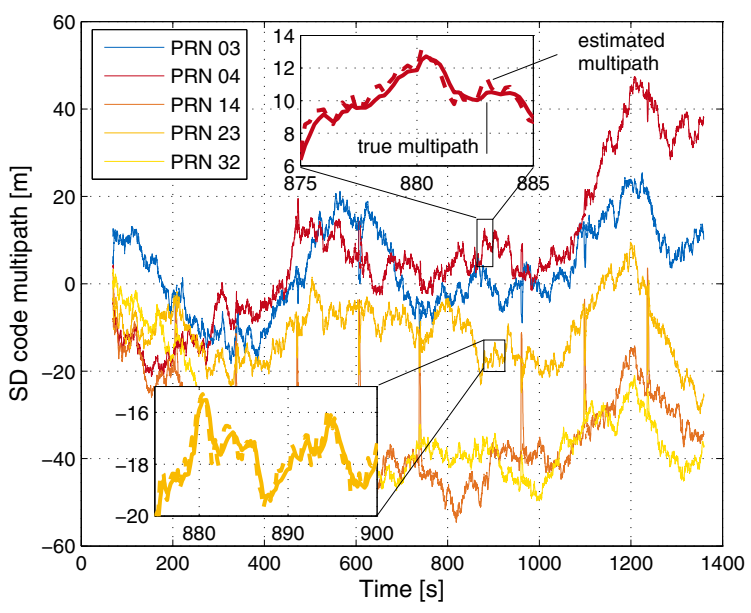

Fig. 2: The estimated multipath closely follows the artifically generated multipath. The error of the multipath estimates is only in the order of the code noise.

Fig. 3 shows the carrier phase residuals of the fixed PPP solution. The residuals of most satellites are in the order of a few millimetres and do not show any long-term drift, which indicates a correct ambiguity resolution. Satellite PRN 19 shows some slight oscillations in the residuals, which indicate phase multipath. The estimated absolute position has an accuracy of a centimeter despite the severe code multipath of up to $50 \mathrm{~m}$.

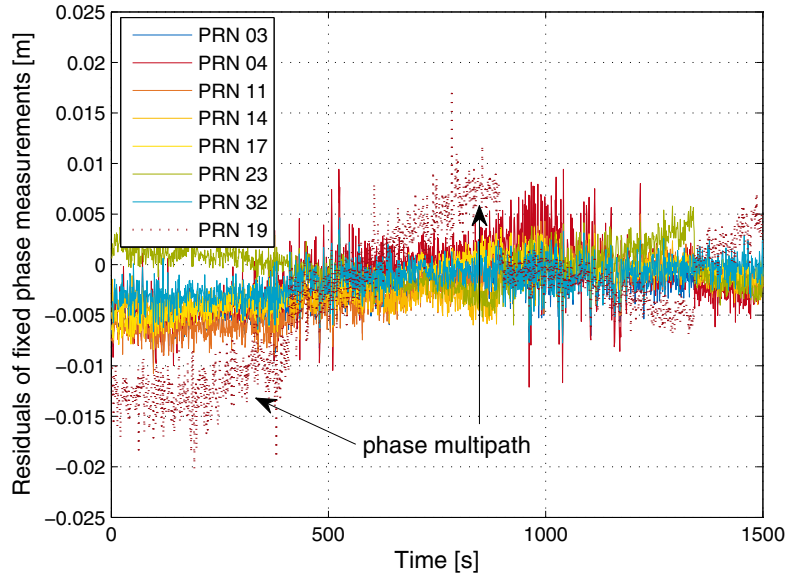

Fig. 3: Residuals of fixed phase measurements

\section{CONCLUSION}

In this paper, we derived a precise model for the pseudorange multipath arising from multiple reflected signals with individual amplitudes, extra-path delays, phase offsets and Doppler shifts. The obtained model was integrated in precise point positioning with GPS/ INS tight coupling. The positioning accuracy was analyzed with low-cost GNSS receivers and SSR corrections and showed a millimeter-level accuracy despite severe code multipath of up to $50 \mathrm{~m}$.

\section{REFERENCES}

[1] P. Teunissen, The least-squares ambiguity decorrelation adjustment: a method for fast GPS integer ambiguity estimation, Journal of Geodesy, pp. 65-82, vol. 70, 1995.

[2] P. Henkel and M. Iafrancesco, Tightly coupled position and attitude determination with two low-cost GNSS receivers, Proc. of 11th International Symposium on Wireless Communications System (ISWCS), pp. 895 900, Barcelona, Spain, 2014.

[3] P. Henkel and A. Sperl, Precise RTK Positioning with GPS/ INS Tight Coupling and Multipath Estimation, Proc. of ION ITM, Monterey, USA, 2016.

[4] B. Parkison, J. Spilker, P. Axelrad and P. Enge, Global Positioning System: Theory and Applications, vol. I and II, AIAA, 1996.

[5] P. Henkel and N. Oku, Cycle slip detection and correction for heading determination with low-cost GPS/ INS receivers, Intern. Assoc. of Geodesy Symposia, Hotine-Marussi Symposium, Rome, Italy, pp. 1-7, 2015.

[6] P. Teunissen, A-PPP: Array-Aided Precise Point Positioning with Global Navigation Satellite Systems, IEEE Transactions on Signal Processing, vol. 60, no. 6, Jun. 2012.

[7] C. Jekeli, Inertial Navigation Systems with Geodetic Applications, 352 pp., Walter de Gruyter, 2001.

[8] P. Henkel and C. Günther, Reliable Integer Ambiguity Resolution: MultiFrequency Code Carrier Linear Combinations and Statistical A Priori Knowledge of Attitude, Navigation, Vol. 59, Nr. 1, pp. 61-75, 2012.

[9] P. Teunissen, Integer least-squares theory for the GNSS compass, Journal of Geodesy, Vol. 84, pp. 433-447, 2010.

[10] P. Henkel and C. Günther, Attitude determination with low-cost GPS/ INS, Proc. of the 26-th Intern. Techn. Meeting of the Instit. of Navigation (ION GNSS+), Nashville, TN, USA, pp. 2015-2023, Sep. 2013.

[11] P. Henkel and J. Kiam, Maximum A Posteriori Probability Estimation of Integer Ambiguities and Baseline, Proc. of 55-th IEEE Symp. ELMAR pp. 353-356, Zadar, Croatia, Sep. 2013.

[12] P. Henkel, Tightly coupled precise point positioning and attitude determination, IEEE Transactions on Aerospace and Electronic Systems, Vol. 51 , iss. 4 , pp. $3182-3197$, Oct. 2015. 